Meta

Journal des traducteurs

Translators' Journal

\title{
Les sociétés professionnelles : vingt ans d'activité
}

\section{Paul A. Horguelin}

Volume 20, numéro 1, mars 1975

Vingt ans de traduction. Bilan et prospectives

URI : https://id.erudit.org/iderudit/003098ar

DOI : https://doi.org/10.7202/003098ar

Aller au sommaire du numéro

Éditeur(s)

Les Presses de l'Université de Montréal

ISSN

0026-0452 (imprimé)

1492-1421 (numérique)

Découvrir la revue

Citer cet article

Horguelin, P. A. (1975). Les sociétés professionnelles : vingt ans d'activité. Meta, 20(1), 75-79. https://doi.org/10.7202/003098ar d'utilisation que vous pouvez consulter en ligne.

https://apropos.erudit.org/fr/usagers/politique-dutilisation/ 


\section{Les sociétés professionnelles: vingt ans d'activité}

Les sociétés professionnelles reflètent, dans une certaine mesure, l'importance, le prestige et le dynamisme des professions dont elles groupent les membres. Aux grandes professions correspondent de grandes associations : ordre des médecins, des avocats, des comptables agréés... Dans cette perspective, l'activité des associations de traducteurs devient un élément important du bilan de la traduction au Canada depuis vingt ans, thème du présent numéro spécial.

Fait assez significatif en ce vingtième anniversaire de notre revue, le premier numéro du Journal des traducteurs publiait un article de J.-P. Vinay sur... l'organisation de la profession. En fait, il s'agissait plutôt d'un regroupement - déjà ! des sociétés de traducteurs, car, comme nous allons le voir, le Canada comptait en 1955 trois associations professionnelles. Mais ce regroupement visait un objectif qui, hélas, n'est pas encore entièrement atteint : rehausser le niveau professionnel en créant un organisme comparable à ce qu'on appelait alors un «Collège », par opposition à une simple association culturelle.

Les traducteurs canadiens avaient senti très tôt le besoin de s'unir. Dès 1920 était fondée l'Association technologique de langue française d'Ottawa, doyenne des associations de traducteurs au Canada et peut-être même dans le monde. En 1943, naissait la Société des traducteurs de Montréal (S.T.M.), regroupant « les traducteurs de carrière et ceux qui s'y destinent, ainsi que ceux qui s'intéressent à l'art de la traduction». En 1954, c'était la création de l'Association des diplômés de l'Institut de traduction de l'Université de Montréal (A.D.I.T.U.M.), qui allait devenir l'année suivante l'Association canadienne des traducteurs diplômés (A.C.T.D.) à qui nous devons la publication du Journal des traducteurs. Ces trois associations semblent œuvrer en harmonie puisqu'elles participent au Congrès des traducteurs tenu le 5 novembre 1955 à Montréal.

Le Journal des traducteurs n'était pas la première publication professionnelle. En 1940, la S.T.M. avait commencé la publication d'une revue, le Traducteur, qui avait comme particularité de publier tous les articles en version bilingue juxtaposée. Après une existence éphémère, cette revue fut remplacée par un bulletin, l'Argus, qui parut jusqu'en 1958 ; on y publiait le compte rendu des activités de la société et des articles sur la langue et la traduction. Enfin, paraissait depuis 1951 le Bulletin de l'Association technologique, principalement orienté vers la terminologie. 
En juin 1956, un groupe de traducteurs de la région d'Ottawa fonde la Société des traducteurs et interprètes du Canada (S.T.I.C.) qui se fixe les buts suivants : 1) grouper les traducteurs et interprètes du Canada en vuc de défendre leurs intérêts à l'échelle nationale et internationale ; 2)' améliorer la qualité de la traduction et de l'interprétation au Canada ; 3 ) favoriser l'étude des deux langues officielles du Canada. La création de cette nouvelle société, à caractère fédératif, va modifier l'échiquier des associations provinciales : en 1957, l'Association technologique se transforme en Société des traducteurs et interprètes d'Ottawa (S.T.I.O.), tandis qu'au Québec est créée la Corporation des traducteurs professionnels du Québec; toutes deux demandent leur affiliation à la S.T.I.C., bientôt suivies par l'Institut de traduction de Montréal. De son côté, la S.T.I.C. adhère à la Fédération internationale des traducteurs (F.I.T.).

La situation devient rapidement confuse. D'une part, la S.T.M. refuse de s'affilier à la nouvelle fédération; d'autre part, certains traducteurs d'Ottawa appartiennent à un troisième groupe : l'Institut professionnel du service public du Canada. On est loin du regroupement envisagé en 1955! Pendant les années qui suivront, la S.T.I.C. déploiera beaucoup d'efforts pour organiser la profession à l'échelle nationale, mais le refus de la S.T.M., principale șociété du Québec, le doublement des activités, notamment en Ontario, et les rivalités que ne pouvait manquer de susciter la multiplication des associations aboutiront à sa disparition en 1970.

La Corporation des traducteurs professionnels du Québec (familièrement appelée «la Corpo »), fondée en avril 1957, va tenter, comme la S.T.I.C. et avec aussi peu de succès, d'être un organisme vraiment professionnel. Elle a pour objet «l'avancement et le rayonnement de la profession de traducteur ainsí que l'étude et la défense des intérêts professionnels, économiques, sociaux et moraux de ses membres ». L'une des conditions d'admission est l'exercice effectif de la profession, ce qui écarte "ceux qui s'intéressent à l'art de la traduction». En pratique, la Corpo restera jusqu'à sa disparition un groupement restreint de traducteurs professionnels.

En 1959, la Société des diplômés de l'Institut de traduction (S.D.I.T.) vient prendre la succession de l'A.C.T.D., apparemment morte de sa belle mort. La S.D.I.T. organisera des séances d'étude et publiera un bulletin, Entre-nous. Elle sera remplacée en juin 1965 par le Cercle des traducteurs, qui élargira son recrutement. C'est aussi en 1965 que la S.T.M. deviendra la Société des traducteurs du Québec (S.T.Q.). La valse des sigles n'est d'ailleurs pas le propre du Québec : en Ontario, la S.T.I.O. s'est muée en A.T.I.O., Association des traducteurs et interprètes de l'Ontario, en septembre 1962.

Pendant que les associations se multiplient ou se transmutent, l'organisation de la profession reste à l'ordre du jour. C'est l'un des thèmes du deuxième congrès des traducteurs et interprètes tenu à l'Université de Montréal en avril 1963. Au cours d'une journée d'étude préliminaire, un conférencier avait décrit les étapes de l'organisation de la profession chez les comptables agréés. La Commission de l'organisation de la profession, après avoir constaté l'impossibilité d'établir un certificat de compétence, pour des raisons.constitutionnelles, fait adopter cinq 
résolutions aux termes desquelles la reconnaissance et l'organisation professionnelles étaient laissées aux sociétés provinciales, à qui on recommandait d'obtenir une loi spéciale de leur parlement respectif, la société nationale assurant la coordination entre les organismes provinciaux. Finalement, un comité constitué de représentants de toutes les associations de traducteurs devait présenter un rapport au congrès suivant... qui n'eut jamais lieu. En Ontario, un comité de l'A.T.I.O. reçut le mandat de rédiger le texte d'un projet de loi, tandis qu'au Québec la Corpo entreprenait des «études préliminaires ». On doit malheureusement constater qu’à partir de ce moment, les rapports entre les associations ontariennes et québécoises vont se relâcher.

Au Québec, il faut attendre 1967 pour assister à un nouveau bond en avant. Sur l'initiative des responsables de l'enseignement de la traduction dans les universités McGill, Laval et de Montréal, un colloque sur l'organisation de la profession se tient à l'Université de Montréal en novembre. Les trois associations de traducteurs (S.T.Q., Cercle et Corpo) y participent. Divers faits avaient préparé le terrain : rapprochement des trois associations, longtemps rivales; sondages officieux indiquant que le législateur souhaitait l'union des traducteurs avant d'envisager leur reconnaissance professionnelle; article dans la Presse où M. J.-M. Laurence préconisait la création d'un «Collège des traducteurs», à l'instar du «Collège des médecins ». Le colloque est un succès. Il s'ouvre sur une déclaration inattendue des présidents des trois associations, qui annoncent la réalisation d'un accord sur le principe d'une fusion dans les cadres de la S.T.Q., et se termine par la création d'un comité de coordination de la profession, chargé de préparer un projet de loi et d'établir les normes professionnelles. Au début de 1968, l'union des traducteurs québécois se réalise au sein de la S.T.Q. L'année 1969 marque le début d'un long cheminement vers la reconnaissance professionnelle, cheminement jalonné d'avatars, d'espoirs déçus, et qui n'est pas encore terminé. Le projet de loi de la S.T.Q. (bill 114) est déposé le 10 avril 1969 ; il prévoit l'exclusivité du titre de «traducteur agréé». Modifié (pour employer un euphémisme) par les conseillers juridiques du gouvernement, redéposé dans sa cinquième version en 1970 , victime d'un changement de gouvernement, il est renvoyé aux calendes grecques par la publication du rapport Castonguay. En 1972, la Commission parlementaire réserve un accueil très favorable aux administrateurs de la S.T.Q. venus plaider leur cause, et en 1973 le Code des professions (loi 250) est sanctionné, ce qui transforme profondément la législation régissant les corporations professionnelles au Québec.

Pendant ce temps, l'A.T.I.O. suivait une voie parallèle en Ontario. Presque en même temps que la S.T.Q., elle avait entrepris des démarches en vue de la reconnaissance professionnelle, mais en demandant l'exclusivité de l'acte. Le dépôt du projet de loi, d'abord prévu pour 1969, fut reporté d'année en année. Comme au Québec, le législateur ontarien entreprit de remettre en cause le processus de la reconnaissance professionnelle, adoptant comme principal critère la protection de l'intérêt public. Pour tenir compte de ce fait nouveau, les deux sociétés provinciales ont dû redéfinir leurs objectifs et modifier radicalement leur approche. Tandis qu'en Ontario l'A.T.I.O. envisage maintenant d'ouvrir la profession à tous ceux qui réussissent l'examen professionnel et de faire une plus grande place au multi- 
linguisme, au Québec, la S.T.Q. demande l'exclusivité du titre de «traducteur agréé » et le monopole de la traduction à la pige.

Si la reconnaissance professionnelle est devenue la priorité des associations du Québec et de l'Ontario, il ne faudrait pas en conclure qu'elle mobilise toutes leurs énergies. Sans attendre le bon vouloir du législateur, ces associations se sont résolument orientées, depuis le début de la présente décennie, vers le statut d'organisme professionnel. Il suffit de passer en revue la liste de leurs comités pour se convaincre de l'étendue de leurs activités, dont rendent compte leurs bulletins d'information : l'Antenne et Informatio.

Les rapports entre les deux grandes sociétés provinciales avaient pris un tournant décisif en 1969, année de la création d'un comité intersociétés qui s'était donné pour tâche de «chercher les moyens d'assurer la collaboration étroite et une saine coordination des deux sociétés afin d'aboutir à l'uniformisation des critères d'admission à l'examen, des normes d'examen et des normes d'exercice de la profession ». Il faut rendre ici hommage au regretté Paul Patenaude qui, par son dynamisme et sa largeur d'esprit, a été l'artisan de ce rapprochement.

En novembre 1970, une autre association voyait le jour: la Corporation des traducteurs et interprètes du Nouveau-Brunswick (C.T.I.N.B.), portant ainsi à trois le nombre des provinces canadiennes où existe une société de traducteurs. Son principal objectif est la reconnaissance de la profession, d'abord par un examen commun avec les autres associations provinciales, et, plus tard, par l'obtention d'une charte provinciale.

À la suite de démarches entreprises par le Comité intersociétés S.T.Q.A.T.I.O., la S.T.I.C. acceptait de disparaître pour laisser la place à une nouvelle fédération, le Conseil des traducteurs et interprètes du Canada (C.T.I.C.), créé à la fin de 1971. Selon ses statuts, le Conseil a pour but: « de coordonner l'activité des sociétés membres et d'encourager leur collaboration; d'assurer l'uniformité des normes relatives à la profession; de maintenir des liens avec les associations nationales et internationales de traducteurs et d'interprètes ». Le C.T.I.C., constitué des trois associations provinciales actuellement existantes (A.T.I.O., S.T.Q., C.T.I.N.B.), s'est affilié à la F.I.T. et y a fait élire un représentant. Il a établi un protocole d'uniformisation des examens professionnels et, à l'échelle internationale, obtenu que le prochain congrès mondial de la F.I.T. se tienne à Montréal en 1977.

En ce début d'année 1975, les traducteurs canadiens sont donc groupés en trois associations provinciales, l'A.T.I.O., la S.T.Q. et la C.T.I.N.B., qui comptent respectivement 375,850 et 26 membres. Sur le plan national et international, ils sont représentés par une fédération, le C.T.I.C. L'organisation de la profession a donc fait des progrès notables depuis vingt ans, les traducteurs ayant réalisé l'unité au sein d'organismes bien structurés et à caractère professionnel.

Quant aux interprètes, ils forment une association commune avec les traducteurs en Ontario et au Nouveau-Brunswick. Les interprètes de l'Ontario sont regroupés au sein d'une section de l'A.T.I.O.; ils ont leurs propres normes d'examen et code de déontologie. Au Québec, les interprètes n'ont pas encore réussi à constituer une association qui pourrait s'affilier au C.T.I.C. Ils sont membres de 
l'A.I.I.C. (Association internationale des interprètes de conférence) et exercent leur profession selon les conditions fixées par le code professionnel de cette association.

Le dernier chapitre de l'organisation de la profession reste à écrire. Il relatera le couronnement des efforts entrepris il y a vingt ans, et poursuivis depuis sans relâche, en vue de faire reconnaître par le législateur le statut professionnel du traducteur. Espérons qu'il ne faudra pas attendre le quarantième anniversaire de Meta pour célébrer cet événement!

Paul-A. Horguelin 